\title{
DEUTERON-PROTON ELASTIC SCATTERING AT INTERMEDIATE ENERGIES
}

\author{
N.B.Ladygina ${ }^{\dagger}$ \\ Laboratory of High Energies, Joint Institute for Nuclear Research, 141980 Dubna, Russia, \\ †E-mail: ladygina@sunhe.jinr.ru
}

\begin{abstract}
The deuteron-proton elastic scattering has been studied in the multiple scattering expansion formalism. The essential attention has been given to such relativistic problem as a deuteron wave function in a moving frame and transformation of spin states due to Wigner rotation. Parameterization of the nucleon-nucleon $t$-matrix has been used to take the off-energy shell effects into account. The vector, $A_{y}$, and tensor, $A_{y y}$, analyzing powers of the deuteron have been calculated at two deuteron kinetic energies: $395 \mathrm{MeV}$ and $1200 \mathrm{MeV}$. The obtained results are compared with the experimental data.
\end{abstract}

PACS: 21.45.+v, 25.45.-z, 25.45.De, 24.10.Jv, 24.70.+s

Keywords: Few-body system, Deuteron-induced reactions, Elastic scattering, Polarization in nuclear reactions

\section{Introduction}

The study of the deuteron-proton elastic scattering has a longtime story both from the experimental [1]- [6] and theoretical [7]- [9] (and refs. therein) points of view. This process is the simplest example of the hadron nucleus collision that is why the interest to this reaction is justified. A number of experiments on deuteron- nucleon elastic scattering is aimed at getting some information about the deuteron wave function as well as nucleon- 
nucleon amplitudes from $N d$ scattering observables. Moreover, the study of the reaction mechanisms, investigations of the few-body scattering dynamics are also very important.

A good theoretical description of the deuteron-nucleon process is obtained for low energies, where the multiple scattering formalism based on the solution of the Faddeev equations has been applied to this problem [7]. Nevertheless, this method requires the realistic nucleon-nucleon potential but the well known $N N$ potentials such as Bonn [10], CD-Bonn [11], Paris [12], Nijmigen [13], AV18 [14], can be used only up to about 300$350 \mathrm{MeV} N N$ laboratory kinetic energy. This fact indicates a necessity to develop new relativistic nucleon-nucleon potentials which describe existing $N N$ data up to $1 \mathrm{GeV}$ and higher. Many efforts to get these kind of $N N$ potentials have been undertaken [15]-[19] but these potentials describe very well only low $N N$ phase shifts. It is possible in the future more acceptable relativistic $N N$ potential will be developed what allows to extend energy region where Faddeev equations formalism can be applied.

At present there are experimental data on the $d p$ elastic scattering in a wide range, where the deuteron kinetic energy is above $400 \mathrm{MeV}$ [1]- [6] (and refs. therein). Already from a few hundred $\mathrm{MeV}$ of nucleon-nucleon interaction energy the relativistic effects begin to play a very important role. A relativistic quantum theory including relativistic kinematics, Lorentz transformation and the relativistic spin theory should be applied to consider a few-body scattering. The basic principles of this theory were formulated in Ref. [20] where results of the works of Dirac [21], Wigner [22], Foldy [23], etc., were developed. In Ref. [24] review devoted to the relativistic dynamics is presented. Recently relativistic kinematics, boost effects and Wigner rotation have been included into the three-nucleon Faddeev equations [25], [26].

The present paper has realized the semi-phenomenological method based on the parameterizations of the deuteron wave function (DWF) and nucleon-nucleon vertices. At intermediate energies the relative momentum of the two nucleons in the deuteron is rather large and, in general, the use of a relativistic deuteron wave function is necessary. In Refs. [27], 28] these DWF's were obtained by solution of the Blankenbecler-Sugar equation. 
But in this approach one of the nucleons is on-energy shell that limits the employment of these DWF's. The two nucleons in the deuteron are considered as the off-energy shell particles in Ref. [29], where the deuteron wave function is the result of the Bethe-Salpeter equation solution. The importance of the relativistic DWF application to describe the deuteron-proton backward elastic scattering was demonstrated in this paper.

However, at the energies considered in the present paper it is possible to use nonrelativistic DWF such as Paris [30], Bonn [10], CD-Bonn [11] due to transition into the deuteron Breit frame. It allows one to decrease the two nucleon relative momentum both in the initial and final deuterons. In this paper the CD-Bonn DWF has been used as a more modern one. It should be noted that the Lorentz transformation and relativistic spin theory have been applied to describe the transformation from the deuteron rest frame to the Breit system. As a result, the deuteron wave function has a more complicated spin structure, than usual nonrelativistic DWF and depends on the two variables. The analogous result was obtained in Refs. [31]-33] for the DWF on the light front.

The model offered in Ref.[8] to investigate the $d p$-elastic scattering at intermediate energies, has been developed further in the present paper. First of all, the deuteron wave function transformation has been done. Secondly, the phase shift analysis data are not used to describe the nucleon-nucleon interaction in comparison with Ref. [8], where the energy dependence in $N N$-vertices important for correct integrations, is lost. Besides, the phase shift analysis gives information about on-energy shell nucleons. But in our method the off-energy shell effects have to be taken into consideration and it can be done by using some parameterization describing the nucleon-nucleon interaction. Thus, the $N N$ $t$-matrix constructed by Love and Franey [34] has been used in our calculations.

The presented method has been applied to calculate two simplest polarization observables: vector, $A_{y}$, and tensor, $A_{y y}$, analyzing powers at two deuteron kinetic energies: 395 $\mathrm{MeV}$ and $1200 \mathrm{MeV}$. The results of these calculations are compared with the experimental data obtained in Saclay [1, 2] and Argonne [3. It should be noted that these observables and energies are examples of the presented method employment. In the future this 
method is planned to describe other polarization observables at deuteron kinetic energies between 400 and $1200 \mathrm{MeV}$.

The paper is organized as follows. Section 2 gives the general formalism: the transformation of the deuteron wave function from the rest frame to a moving deuteron system, is considered; the description of the nucleon-nucleon interaction is presented; the calculations of the scattering amplitude terms are performed. Section 3 defines the polarization observables, $A_{y}$ and $A_{y y}$. The results of the calculations are discussed in Sect.4. The conclusions are contained in Sect.5.

\section{General formalism}

According to the three-body collision theory, the amplitude of the deuteron-proton elastic scattering $\mathcal{J}$ is defined by the matrix element of the transition operator $U_{11}$ :

$$
U_{d p \rightarrow d p}=\delta\left(E_{d}+E_{p}-E_{d}^{\prime}-E_{p}^{\prime}\right) \mathcal{J}=<1(23)\left|\left[1-P_{12}-P_{13}\right] U_{11}\right| 1(23)>\quad .
$$

Here the state $\mid 1(23)>$ corresponds to the configuration, when nucleons 2 and 3 form the deuteron state and nucleon 1 is free. Appearance of the permutation operators for two nucleons $P_{i j}$ reflects the fact that the initial and final states are antisymmetric due to exchange of the two particles.

The transition operators for rearrangement scattering are defined by the Alt-GrassbergerSandhas equations [35], 36]

$$
\begin{array}{lll}
U_{11} & = & t_{2} g_{0} U_{21}+t_{3} g_{0} U_{31} \\
U_{21} & = & g_{0}^{-1}+t_{1} g_{0} U_{11}+t_{3} g_{0} U_{31} \\
U_{31} & = & g_{0}^{-1}+t_{1} g_{0} U_{11}+t_{2} g_{0} U_{21},
\end{array}
$$

where $t_{1}=t(2,3)$,etc., is the t-matrix of the two-nucleon interaction and $g_{0}$ is the free three-particle propagator. The indices $i j$ for the transition operators $U_{i j}$ denote free particles $i$ and $j$ in the final and initial states, respectively.

Iterating these equations up to the $t_{i}$ second order terms we can present the reaction amplitude as a sum of the following three contributions: one nucleon exchange, single 
scattering and double scattering terms, -

$$
\begin{aligned}
\mathcal{J}_{d p \rightarrow d p} & =\mathcal{J}_{\text {ONE }}+\mathcal{J}_{S S}+\mathcal{J}_{D S} \\
\mathcal{J}_{O N E} & =-2<1(23)\left|P_{12} g_{0}^{-1}\right| 1(23)> \\
\mathcal{J}_{S S} & =2<1(23)\left|t_{3}^{\text {sym }}\right| 1(23)> \\
\mathcal{J}_{D S} & =2<1(23)\left|t_{3}^{\text {sym }} g_{0} t_{2}^{\text {sym }}\right| 1(23)>,
\end{aligned}
$$

where we have introduced notations for antisymmetrized operators $t_{2}^{\text {sym }}=\left[1-P_{13}\right] t_{2}$ and $t_{3}^{\text {sym }}=\left[1-P_{12}\right] t_{3}$. Before we consider each of these elements in detail, we must determine the reference frame.

\section{Breit system}

The most popular deuteron wave functions are well known only in the nonrelativistic region. In this connection it is very important to choose the frame, where the application of the nonrelativistic deuteron wave function is possible. The frame which minimizes the relative momenta of the nucleons in the both deuteron wave functions, is the deuteron Breit system. In this frame the deuterons move in opposite directions with equal momenta (fig.1). As a consequence, the energies of the initial and final deuterons (and protons) are equal to each other.

$$
\begin{aligned}
& E_{d}=E_{d}^{\prime}=\sqrt{M_{d}^{2}+\vec{Q}^{2}}, \quad E_{p}=E_{p}^{\prime}=\sqrt{m^{2}+\vec{p}^{2}} \\
& (\vec{p} \vec{Q})=-\vec{Q}^{2}
\end{aligned}
$$

We take the orthonormal basis

$$
\vec{z}=\frac{\vec{p}-\vec{p}^{\prime}}{\left|\vec{p}-\vec{p}^{\prime}\right|}=-\hat{Q}, \quad \vec{x}=\frac{\vec{p}+\vec{p}^{\prime}}{\left|\vec{p}+\vec{p}^{\prime}\right|}=\hat{p+Q}, \quad \vec{y}=\vec{z} \times \vec{x}=\hat{p} \times \hat{Q}
$$

where $z$-axis goes along the transfer momentum and $x$-axis - along the average momentum of the initial and final protons. As usual, the $y$-axis is normal to the scattering plane. 


\section{Deuteron wave function}

In order to get the wave function of the moving deuteron, let us consider an arbitrary frame where two nucleons have momenta $\vec{p}_{1}, \vec{p}_{2}$ and energies $E_{1}=\sqrt{m_{N}^{2}+\vec{p}_{1}^{2}}, E_{2}=$ $\sqrt{m_{N}^{2}+{\overrightarrow{p_{2}}}^{2}}$, respectively. Here $m_{N}$ is the nucleon mass. These momenta are related with those in the center-of-mass (c.m.) by Lorenz transformation

$$
\begin{aligned}
& L(\vec{u}) p_{1}=\left(E^{*}, \vec{p}\right) \\
& L(\vec{u}) p_{2}=\left(E^{*},-\vec{p}\right)
\end{aligned}
$$

with velocity

$$
\vec{u}=\frac{\vec{p}_{1}+\vec{p}_{2}}{E_{1}+E_{2}}
$$

Here the c.m. energy of one of the nucleons $E^{*}$ is related with Mandelstam variable $s$ by

$$
E^{*}=\sqrt{s} / 2
$$

Thus, we can introduce new variables $\vec{Q}$ and $\vec{k}$ which can be expressed through $\vec{p}_{1}$ and $\vec{p}_{2}$

$$
\begin{aligned}
& \vec{Q}=\vec{p}_{1}+\vec{p}_{2} \\
& \vec{k}=\frac{\left(E_{2}+E^{*}\right) \vec{p}_{1}-\left(E_{1}+E^{*}\right) \vec{p}_{2}}{E_{1}+E_{2}+2 E^{*}} .
\end{aligned}
$$

Then a two-nucleon state in the $\left(\vec{p}_{1}, \vec{p}_{2}\right)$ system is connected with a two-nucleon state in the center-of-mass by the relation

$$
\left|\vec{p}_{1}, \vec{p}_{2}>=J^{-1 / 2}\left(\vec{p}_{1}, \vec{p}_{2}\right) W_{1 / 2}\left(\vec{p}_{1}, \vec{u}\right) W_{1 / 2}\left(\vec{p}_{2}, \vec{u}\right)\right| \vec{k}, \vec{Q}>
$$

where $\vec{k}$ is the relative momentum of the two nucleons in the c.m. The normalization factor provides conditions of orthonormality and completeness and is defined by the Jacobian of the transformations [20]

$$
J\left(\vec{p}_{1}, \vec{p}_{2}\right)=\frac{\partial\left(\vec{p}_{1}, \vec{p}_{2}\right)}{\partial(\vec{k}, \vec{Q})}=\frac{2}{E^{*}} \frac{E_{1} E_{2}}{E_{1}+E_{2}} .
$$

The Wigner rotation operator in the spin space of the $i$-th nucleon has the standard form

$$
W_{1 / 2}\left(\vec{p}_{i}, \vec{u}\right)=\exp \left\{-i \omega_{i}\left(\vec{n}_{i} \vec{\sigma}_{i}\right) / 2\right\}=\cos \left(\omega_{i} / 2\right)\left[1-i\left(\vec{n}_{i} \vec{\sigma}_{i}\right) \operatorname{tg}\left(\omega_{i} / 2\right)\right]
$$


with the rotation axis

$$
\vec{n}_{i}=\frac{\vec{u} \times \vec{p}_{i}}{\left|\vec{u} \times \vec{p}_{i}\right|}
$$

Ref.[20] shows that under a pure Lorenz transformation

$$
L\left(\vec{u}^{\prime}\right) \vec{P}=\vec{Q}
$$

the wave function of the bound state transforms in the same way as the state of a single particle

$$
W\left(L_{\vec{u}^{\prime}}\right)\left|\vec{P}>=\sqrt{\frac{E_{\vec{Q}}}{E_{\vec{P}}}} W_{1}\left(\vec{Q}, \vec{u}^{\prime}\right)\right| \vec{Q}>,
$$

where the $W_{1}$ is the Wigner rotation operator for spin 1 particle

$$
W_{1}\left(\vec{Q}, \vec{u}^{\prime}\right)=\exp \left\{-i \omega\left(\vec{n}^{\prime} \vec{S}\right)\right\}
$$

with the rotation axis

$$
\vec{n}^{\prime}=\frac{\vec{u}^{\prime} \times \vec{Q}}{\left|\vec{u}^{\prime} \times \vec{Q}\right|} .
$$

The deuteron wave function in the rest frame of the deuteron depends on only one variable $\vec{k}$, which is the relative momentum of the outgoing proton and neutron

$$
<m_{p} m_{n}\left|\Omega_{d}\right| \mathcal{M}_{d}>=\frac{1}{\sqrt{4 \pi}}<m_{p} m_{n}\left|\left\{u(k)+\frac{w(k)}{\sqrt{8}}\left[3\left(\vec{\sigma}_{1} \hat{k}\right)\left(\vec{\sigma}_{2} \hat{k}\right)-\left(\vec{\sigma}_{1} \vec{\sigma}_{2}\right)\right]\right\}\right| \mathcal{M}_{d}>
$$

where $u(k)$ and $w(k)$ describe the $S$ and $D$ components of the deuteron wave function [10], [11, [30], $\hat{k}$ is the unit vector in $\vec{k}$ direction and $\mathcal{M}_{d}, m_{p}, m_{n}$ are spinors of the deuteron, proton, and neutron, respectively.

Obviously, the deuteron rest frame corresponds to the center-of-mass of the two outgoing nucleons, $\vec{P}=\vec{p}_{n}+\vec{p}_{p}=\overrightarrow{0}$. Then the proton -neutron relative momentum $\vec{k}$ in Eq.(18) is related with the nucleon momenta $\vec{p}_{1}$ and $\vec{p}_{2}$ in the arbitrary frame by Eq.(9). Using the formulae (91)-(18) we get the expression for the deuteron wave function in the frame, where the deuteron is moving and its momentum is $\vec{Q}$

$$
\begin{aligned}
<\vec{p}_{1} \vec{p}_{2}, m_{1} m_{2}\left|\Omega_{d}\right| \vec{Q}, \mathcal{M}_{d}> & =\sqrt{\frac{M_{d}}{E_{\vec{Q}}}} \sqrt{\frac{E^{*}\left(E_{1}+E_{2}\right)}{2 E_{1} E_{2}}} \cos \frac{\omega_{1}}{2} \cos \frac{\omega_{2}}{2} . \\
& <\vec{k} \vec{Q}, m_{1}^{\prime} m_{2}^{\prime}\left|\left[1-i\left(\vec{n} \vec{\sigma}_{1}\right) \operatorname{tg} \frac{\omega_{1}}{2}\right]\left[1+i\left(\vec{n} \vec{\sigma}_{2}\right) \operatorname{tg} \frac{\omega_{2}}{2}\right] \Omega_{d}\right| \overrightarrow{0}, \mathcal{M}_{d}>.
\end{aligned}
$$


The Wigner rotation is performed around the normal to the $(\vec{k} \vec{Q})$ plane

$$
\vec{n}=\frac{\vec{p}_{1} \times \vec{p}_{2}}{\left|\overrightarrow{p_{1}} \times \overrightarrow{p_{2}}\right|}=\frac{\vec{k} \times \vec{Q}}{|\vec{k} \times \vec{Q}|}
$$

on the rotation angles, which are defined by the relations [37]:

$$
\begin{aligned}
\operatorname{tg} \frac{\omega_{1}}{2} & =\frac{\left|\vec{p}_{1} \times \vec{p}_{2}\right|}{m_{N}\left(E_{1}+E_{2}+2 E^{*}\right)+2 E^{*}\left(E_{1}+E^{*}\right)} \\
\operatorname{tg} \frac{\omega_{2}}{2} & =\frac{\left|\vec{p}_{1} \times \vec{p}_{2}\right|}{m_{N}\left(E_{1}+E_{2}+2 E^{*}\right)+2 E^{*}\left(E_{2}+E^{*}\right)} .
\end{aligned}
$$

The expression (19) can be presented through six terms:

$$
\begin{aligned}
<\vec{p}_{1} \vec{p}_{2}, m_{1} m_{2}\left|\Omega_{d}\right| \vec{Q}, \mathcal{M}_{d}> & =g_{1}(\vec{k}, \vec{Q})+g_{2}(\vec{k}, \vec{Q})\left(\vec{\sigma}_{1} \vec{n}\right)\left(\vec{\sigma}_{2} \vec{n}\right)+g_{3}(\vec{k}, \vec{Q})\left(\vec{\sigma}_{1} \vec{\sigma}_{2}\right)+ \\
& +g_{4}(\vec{k}, \vec{Q})\left(\vec{\sigma}_{1} \hat{k}\right)\left(\vec{\sigma}_{2} \hat{k}\right)+g_{5}(\vec{k}, \vec{Q})\left[\left(\vec{\sigma}_{1}+\vec{\sigma}_{2}\right) \vec{n}\right]+ \\
& +g_{6}(\vec{k}, \vec{Q})\left[\left(\vec{\sigma}_{1} \hat{k}\right)\left(\vec{\sigma}_{2} \vec{n} \times \hat{k}\right)+\left(\vec{\sigma}_{1} \vec{n} \times \hat{k}\right)\left(\vec{\sigma}_{2} \hat{k}\right)\right] .
\end{aligned}
$$

Solving a relativistic equation one can obtain functions $g_{i}$ as it was done in Ref.[31][33] on the light front. But in this paper a usual nonrelativistic DWF is taken as input. Therefore, functions $g_{i}$ are defined as linear combinations of $u$ and $w$ (S-and $D$-waves).

This way we have got the relation between the deuteron wave function in two systems: the deuteron rest frame and the arbitrary moving system. Note, the Wigner rotation corrections are small at the energies under consideration, but momenta transformations are very important since they give us an opportunity to use the nonrelativistic deuteron wave functions in a rather wide energy region.

\section{One nucleon exchange scattering}

The first contribution into the $d p$-elastic scattering amplitude $\mathcal{J}$ in Eq.(3) is the one nucleon exchange (ONE) term. The corresponding diagram is presented in Fig.1a. Applying the definitions of the wave function of a moving deuteron and three- nucleon free propagator, we can write ONE amplitude in the following form:

$$
\begin{aligned}
\mathcal{J}_{O N E}=-2 & { }_{1(23)}<\vec{p}^{\prime} m^{\prime} \tau^{\prime} ;-\vec{Q} \mathcal{M}_{d}^{\prime} 0 \mid \Omega_{d}^{\dagger}(23) P_{12} \\
& \left(E_{d}+E_{p}-\hat{K}_{1}-\hat{K}_{2}-\hat{K}_{3}+i \varepsilon\right) \Omega_{d}(23) \mid \vec{Q} \mathcal{M}_{d} 0 ; \vec{p} m \tau>_{1(23)},
\end{aligned}
$$


where $m, m^{\prime}$ are the spin projections of the initial and final protons, $\tau, \tau^{\prime}$ are their isospin projections, respectively. The kinetic-energy operator has the standard definition, $\hat{K}_{i}\left|\vec{p}_{i}>=\sqrt{m_{N}^{2}+\vec{p}_{i}^{2}}\right| \vec{p}_{i}>$.

After a straightforward calculation we have the expression for the ONE amplitude

$$
\begin{aligned}
\mathcal{J}_{O N E}= & -\frac{1}{2}\left(E_{d}-E_{p}-\sqrt{m_{N}^{2}+\vec{p}^{2}-\vec{Q}^{2}}\right) . \\
& <\vec{p}^{\prime} m^{\prime} ;-\vec{Q} \mathcal{M}_{d}^{\prime}\left|\Omega_{d}^{\dagger}(23)\left[1+\left(\vec{\sigma}_{1} \vec{\sigma}_{2}\right)\right] \Omega_{d}(23)\right| \vec{Q} \mathcal{M}_{d} ; \overrightarrow{p m}>,
\end{aligned}
$$

where the definition of the permutation operator in spin space $P_{12}(\sigma)=\frac{1}{2}\left[1+\left(\vec{\sigma}_{1} \vec{\sigma}_{2}\right)\right]$ has been applied. The two nucleons relative momentum in the deuteron rest frame is the argument of the deuteron wave function in Eq.(18). Using Eq.(9) we can express these variables for the initial $\vec{p}_{0}$ and final $\vec{p}_{0}^{\prime}$ deuterons, correspondingly,

$$
\begin{aligned}
& \vec{p}_{0}=\vec{p}+\vec{Q}\left[1+\frac{E_{n}+E^{*}}{E_{p}+E_{n}+E^{*}}\right] \\
& \vec{p}_{0}^{\prime}=\vec{p}+\vec{Q}\left[1-\frac{E_{n}+E^{*}}{E_{p}+E_{n}+E^{*}}\right] .
\end{aligned}
$$

Here $E_{n}=\sqrt{m_{N}^{2}+\vec{p}^{2}-\vec{Q}^{2}}$ and $E^{*}=\sqrt{\left(E_{p}+E_{n}\right)^{2}-\vec{Q}^{2}} / 2$ are the struck neutron energy in the moving deuteron frame and rest deuteron frame, respectively. Note, that $\left|\vec{p}_{0}\right|=\left|\vec{p}_{0}^{\prime}\right|$. The Wigner rotations are performed around $y$-axis on the equal angles both for the initial and final deuterons. Using Eq.(21) we get

$$
\begin{gathered}
\operatorname{tg} \frac{\omega_{1}}{2}=\operatorname{tg} \frac{\omega_{1}^{\prime}}{2}=\frac{|\vec{p} \times \vec{Q}|}{m_{N}\left(E_{p}+E_{n}+2 E^{*}\right)+2 E^{*}\left(E_{p}+E^{*}\right)} \\
\operatorname{tg} \frac{\omega_{2}}{2}=\operatorname{tg} \frac{\omega_{2}^{\prime}}{2}=\frac{|\vec{p} \times \vec{Q}|}{m_{N}\left(E_{p}+E_{n}+2 E^{*}\right)+2 E^{*}\left(E_{n}+E^{*}\right)}
\end{gathered}
$$

where the angles $\omega_{1}, \omega_{1}^{\prime}$ are related with the projectile and outgoing protons, respectively, while $\omega_{2}, \omega_{2}^{\prime}$ correspond to the struck neutron (Fig.1a).

\section{Single scattering}

The other term in the $d p$-elastic scattering amplitude Eq.(3) is the single scattering one. The corresponding diagram is presented in Fig.(1b). Following the standard procedure and applying the definition of the moving deuteron wave function Eq.(19), we get 
the expression for the single scattering amplitude:

$$
\begin{aligned}
\mathcal{J}_{S S} & =\int d \vec{q}^{\prime}<-\vec{Q} \mathcal{M}_{d}^{\prime}\left|\Omega_{d}^{\dagger}\right| \vec{q}^{\prime} m^{\prime \prime},-\vec{Q}-\vec{q}^{\prime} m_{3}^{\prime}> \\
& <\vec{p}^{\prime} m^{\prime},-\vec{Q}-\vec{q}^{\prime}\left|\frac{3}{2} t_{12}^{1}+\frac{1}{2} t_{12}^{0}\right| \vec{p} m, \vec{Q}-\vec{q}^{\prime} m_{2}^{\prime}><\vec{q}^{\prime} m^{\prime \prime}, \vec{Q}-\vec{q}^{\prime} m_{2}^{\prime}\left|\Omega_{d}\right| \vec{Q} \mathcal{M}_{d}>
\end{aligned}
$$

The relative momenta of two nucleons for the initial and final deuterons are

$$
\begin{aligned}
& \vec{p}_{0}=\vec{q}^{\prime}-\vec{Q} \frac{E_{2}+E^{*}}{E_{2}+E_{3}+2 E^{*}} \\
& \vec{p}_{0}^{\prime}=\vec{q}^{\prime}+\vec{Q} \frac{E_{2}+E^{\prime *}}{E_{2}+E_{3}^{\prime}+2 E^{\prime *}},
\end{aligned}
$$

where the nucleons energies $E_{2}, E_{3}, E_{3}^{\prime}$ in the reference frame are defined by the standard manner (Fig.1b)

$$
E_{2}=\sqrt{m_{N}^{2}+\vec{q}^{\prime 2}}, \quad E_{3}=\sqrt{m_{N}^{2}+\left(\vec{Q}-\vec{q}^{\prime}\right)^{2}}, \quad E_{3}^{\prime}=\sqrt{m_{N}^{2}+\left(\vec{Q}+\vec{q}^{\prime}\right)^{2}}
$$

and these energies in the center-of-mass of the two nucleons forming the initial and final deuterons are equal, correspondingly, to

$$
E^{*}=\frac{1}{2} \sqrt{\left(E_{2}+E_{3}\right)^{2}-\vec{Q}^{2}}, \quad E^{*}=\frac{1}{2} \sqrt{\left(E_{2}+E_{3}^{\prime}\right)^{2}-\vec{Q}^{2}} .
$$

The Wigner rotation axis goes along the normal to $\left(\vec{q}^{\prime} \vec{Q}\right)$ plane

$$
\vec{n}=\frac{\vec{q}^{\prime} \times \vec{Q}}{\left|\vec{q}^{\prime} \times \vec{Q}\right|}
$$

and Wigner rotation angles are defined in accordance with Eq.(21) for the initial

$$
\begin{aligned}
\operatorname{tg} \frac{\omega_{2}}{2} & =\frac{\left|\vec{q}^{\prime} \times \vec{Q}\right|}{m_{N}\left(E_{2}+E_{3}+2 E^{*}\right)+2 E^{*}\left(E_{2}+E^{*}\right)} \\
\operatorname{tg} \frac{\omega_{3}}{2} & =-\frac{\left|\vec{q}^{\prime} \times \vec{Q}\right|}{m_{N}\left(E_{2}+E_{3}+2 E^{*}\right)+2 E^{*}\left(E_{3}+E^{*}\right)}
\end{aligned}
$$

and final deuterons

$$
\begin{aligned}
\operatorname{tg} \frac{\omega_{2}^{\prime}}{2} & =-\frac{\left|\vec{q}^{\prime} \times \vec{Q}\right|}{m_{N}\left(E_{2}+E_{3}^{\prime}+2 E^{\prime *}\right)+2 E^{\prime *}\left(E_{2}+E^{\prime *}\right)} \\
\operatorname{tg} \frac{\omega_{3}^{\prime}}{2} & =\frac{\left|\vec{q}^{\prime} \times \vec{Q}\right|}{m_{N}\left(E_{2}+E_{3}^{\prime}+2 E^{\prime *}\right)+2 E^{\prime *}\left(E_{3}^{\prime}+E^{\prime *}\right)} .
\end{aligned}
$$


Further we have to give essential attention to the two nucleon $t^{i}$-matrix in Eq.(27). Here index $i$ denotes the isospin state of the nucleon-nucleon system. This $t$-matrix is the half-off-energy shell one. Since we assume to use the formalism presented here to investigate the processes at rather high energies, we can not define the high-energy $N N$ t-matrix solving the Lippmann-Schwinger equation with any known potential. In this situation it is possible to apply some parameterization as in Refs. 38], [39] to study the $d p$ breakup in the $\mathrm{GeV}$ region.

\section{Nucleon-nucleon $t$-matrix}

In order to describe the nucleon- nucleon interaction in a wide energy region, we use the Love and Franey parameterization [34]. In this model the on-shell $N N t$-matrix is defined in the center-of-mass

$$
\begin{array}{r}
<\kappa^{\prime} m_{1}^{\prime} m_{2}^{\prime}|t| \kappa m_{1} m_{2}>=<\vec{\kappa}^{\prime} m_{1}^{\prime} m_{2}^{\prime} \mid A+B\left(\vec{\sigma}_{1} \hat{N}^{*}\right)\left(\vec{\sigma}_{2} \hat{N}^{*}\right)+C\left(\vec{\sigma}_{1}+\vec{\sigma}_{2}\right) \cdot \hat{N}^{*}+ \\
D\left(\vec{\sigma}_{1} \hat{q}^{*}\right)\left(\vec{\sigma}_{2} \hat{q}^{*}\right)+F\left(\vec{\sigma}_{1} \hat{Q}^{*}\right)\left(\vec{\sigma}_{2} \hat{Q}^{*}\right) \mid \vec{\kappa} m_{1} m_{2}>,
\end{array}
$$

where the orthonormal basis is combinations of the nucleons relative momenta in the initial $\vec{\kappa}$ and final $\vec{\kappa}^{\prime}$ states

$$
\hat{q}^{*}=\frac{\vec{\kappa}-\vec{\kappa}^{\prime}}{\left|\vec{\kappa}-\vec{\kappa}^{\prime}\right|} \quad, \quad \hat{Q}^{*}=\frac{\vec{\kappa}+\vec{\kappa}^{\prime}}{\left|\vec{\kappa}+\vec{\kappa}^{\prime}\right|} \quad, \quad \hat{N}^{*}=\frac{\vec{\kappa} \times \vec{\kappa}^{\prime}}{\left|\vec{\kappa} \times \vec{\kappa}^{\prime}\right|}
$$

We can not directly use this definition of the $t$-matrix in our calculation. First of all, because this $t$-matrix is defined in the c.m., while we need that in the deuteron Breit frame. Second, we need the off-energy shell $t$-matrix, but the Love and Franey parameterization gives us the on-energy shell matrix. However, the both problems can be solved.

Using formulae (10)-(13) we can connect expressions for the $N N$-matrix in the c.m. with the same matrix in the reference frame by the following relation:

$$
<\vec{p}^{\prime} \vec{p}_{3}^{\prime} ; m^{\prime} m_{3}^{\prime}|t| \vec{p} \vec{p}_{3} ; m m_{3}>=\mathcal{N}<\kappa^{\prime} m_{1}^{\prime} m_{2}^{\prime}\left|W_{1 / 2}^{\dagger}\left(\vec{p}^{\prime}\right) W_{1 / 2}^{\dagger}\left(\vec{p}_{3}^{\prime}\right) t W_{1 / 2}(\vec{p}) W_{1 / 2}\left(\vec{p}_{3}\right)\right| \kappa m_{1} m_{2}>
$$

Here momentum notations correspond to the ones given in Fig.1b. The Wigner rotations 
of the initial and final states are performed around $\vec{\mu}$ and $\vec{\mu}^{\prime}$-axes, respectively:

$$
\vec{\mu}=\frac{\vec{p} \times \vec{p}_{3}}{\left|\vec{p} \times \vec{p}_{3}\right|}, \quad \vec{\mu}^{\prime}=\frac{\vec{p}^{\prime} \times \vec{p}_{3}^{\prime}}{\left|\vec{p}^{\prime} \times \vec{p}_{3}^{\prime}\right|}
$$

We introduce the following notations for the Wigner angles: $\chi_{1}, \chi_{1}^{\prime}$ correspond to the projectile and outgoing protons, while $\chi_{3}, \chi_{3}^{\prime}$ are related with the nucleons from the initial and final deuterons, correspondingly. These angles can be expressed through their tangents using Eq.(21):

$$
\begin{aligned}
\operatorname{tg} \frac{\chi_{1}}{2} & =\frac{\left|\vec{p} \times \vec{p}_{3}\right|}{m_{N}\left(E_{p}+E_{3}+2 E_{13}^{*}\right)+2 E_{13}^{*}\left(E_{p}+E_{13}^{*}\right)} \\
\operatorname{tg} \frac{\chi_{3}}{2} & =-\frac{\left|\vec{p} \times \vec{p}_{3}\right|}{m_{N}\left(E_{p}+E_{3}+2 E_{13}^{*}\right)+2 E_{13}^{*}\left(E_{3}+E_{13}^{*}\right)} \\
\operatorname{tg} \frac{\chi_{1}^{\prime}}{2} & =\frac{\left|\vec{p}^{\prime} \times \vec{p}_{3}^{\prime}\right|}{m_{N}\left(E_{p}+E_{3}^{\prime}+2 E_{13}^{* \prime}\right)+2 E_{13}^{* \prime}\left(E_{p}+E_{13}^{* \prime}\right)} \\
\operatorname{tg} \frac{\chi_{3}^{\prime}}{2} & =-\frac{\left|\vec{p}^{\prime} \times \vec{p}_{3}^{\prime}\right|}{m_{N}\left(E_{p}+E_{3}^{\prime}+2 E_{13}^{* \prime}\right)+2 E_{13}^{* \prime}\left(E_{3}^{\prime}+E_{13}^{* \prime}\right)} .
\end{aligned}
$$

As it is shown in ref. [8] the spin rotation corrections to the $N N t$ - matrix are very small at the nucleon kinetic energy of $800 \mathrm{MeV}$. Therefore in our calculations we neglect the second term in Eq.(12). Thus, only cosines of the Wigner angles are the Wigner operators in Eq.(35) and, therefore, the $N N$ - matrix in the reference frame has the same spin structure as that in the c.m.

The normalization factor in Eq.(35) is related with the Jacobian of the transformations Eq.(11) and equals to

$$
\mathcal{N}=\sqrt{\frac{E_{13}^{*}\left(E_{p}+E_{3}\right)}{2 E_{p} E_{3}}} \sqrt{\frac{E_{13}^{* \prime}\left(E_{p}+E_{3}^{\prime}\right)}{2 E_{p} E_{3}^{\prime}}},
$$

where $E_{13}^{*}$ and $E_{13}^{\prime *}$ are the initial and final c.m. energies of the two nucleons taking part in the interaction, respectively:

$$
E_{13}^{*}=\frac{1}{2} \sqrt{\left(E_{p}+E_{3}\right)^{2}-\vec{K}^{2}}, \quad E_{13}^{* \prime}=\frac{1}{2} \sqrt{\left(E_{p}+E_{3}^{\prime}\right)^{2}-\vec{K}^{2}}
$$

with a full momentum

$$
\vec{K}=\vec{p}+\vec{Q}-\vec{q}^{\prime}
$$


In our calculations we need the half-off-energy shell and off-energy shell nucleonnucleon $t$-matrices, while Eq.(34) gives us only the on-energy shell matrix. In order to reach this purpose, we use the result of the Ref. [40] which shows the off-energy shell t-matrix to be connected with the on-energy shell matrix by the relation

$$
t\left(E_{\text {onshell }} ; \vec{K} ; \vec{\kappa}^{\prime} \vec{\kappa}\right)=F t\left(\sqrt{s} ; \vec{\kappa}^{\prime} \vec{\kappa}\right)
$$

where the kinematical factor $F$ is defined as

$$
F=\frac{E_{\text {onshell }}}{\sqrt{s}} \frac{\sqrt{s}+2 E^{*}}{E_{\text {onshell }}+E_{p}+E_{3}} \frac{\sqrt{s}+2 E^{\prime *}}{E_{\text {onshell }}+E_{p}+E_{3}^{\prime}} .
$$

Here $E_{\text {onshell }}$ denotes the energy, which is determined by the following condition:

$$
E_{\text {onshell }}=E_{p}+E_{3}=E_{p}+E_{3}^{\prime}
$$

For the single scattering this condition will be satisfied at $\left(\vec{Q} \vec{q}^{\prime}\right)=0$. Then, $E_{\text {onshell }}=$ $E_{p}+\sqrt{m_{N}^{2}+\vec{Q}^{2}+\vec{q}^{\prime 2}}$.

The argument of the on-energy shell $t$-matrix in Eq.(40) is the square root of the Mandelstam variable $s$ :

$$
s=E_{\text {onshell }}^{2}-\vec{K}^{2}
$$

Thus, using the scenario presented here we can extend the parameterized on-energy shell $N N$ t-matrix to the off- energy shell case.

\section{Double scattering}

The diagram of the double scattering is shown in Fig.(1c). The corresponding amplitude $\mathcal{J}_{D S}$ can be written as

$$
\begin{aligned}
\mathcal{J}_{D S}= & N N^{\prime} \int d \vec{q}^{\prime} \int d \vec{q}^{\prime \prime}<-\vec{Q} \mathcal{M}_{d}^{\prime}\left|\Omega_{d}^{\dagger}\right| \vec{q}^{\prime} m_{2}^{\prime}, \vec{Q}-\vec{q}^{\prime} m_{3}^{\prime}> \\
& <m^{\prime} m_{2}^{\prime} m_{3}^{\prime} \mid\left\{t_{12}^{1}\left(\sqrt{s_{12}^{\prime}}, \vec{\kappa}, \vec{\kappa}^{\prime}\right) t_{13}^{1}\left(\sqrt{s_{13}}, \vec{k}, \vec{k}^{\prime}\right)+\right. \\
& \left.\frac{1}{4}\left[t_{12}^{1}\left(\sqrt{s_{12}^{\prime}}, \vec{\kappa}, \vec{\kappa}^{\prime}\right)+t_{12}^{0}\left(\sqrt{s_{12}^{\prime}}, \vec{\kappa}, \vec{\kappa}^{\prime}\right)\right]\left[t_{13}^{1}\left(\sqrt{s_{13}}, \vec{k}, \vec{k}^{\prime}\right)+t_{13}^{0}\left(\sqrt{s_{13}}, \vec{k}, \vec{k}^{\prime}\right)\right]\right\} \\
& \frac{1}{E_{d}+E_{p}-E_{1}^{\prime}-E_{2}^{\prime}-E_{3}^{\prime}+i \varepsilon}\left|m m_{2} m_{3}><-\vec{Q}-\vec{q}^{\prime \prime} m_{2}, \vec{q}^{\prime \prime} m_{3}\right| \Omega_{d} \mid \mathcal{M}_{d}>.
\end{aligned}
$$


As before, we define the relative momenta of the nucleons which form the initial and final deuterons, respectively, in accordance with Eq.(9)

$$
\begin{aligned}
\vec{p}_{0} & =\vec{q}^{\prime}-\vec{Q} \frac{E_{2}+E^{*}}{E_{2}+E_{3}+2 E^{*}} \\
\vec{p}_{0}^{\prime} & =-\vec{q}^{\prime \prime}-\vec{Q} \frac{E_{3}^{\prime}+E^{\prime *}}{E_{2}^{\prime}+E_{3}^{\prime}+2 E^{\prime *}}
\end{aligned}
$$

where the center-of-mass energies of these nucleons are related with those in the reference frame by

$$
E^{*}=\frac{1}{2} \sqrt{\left(E_{2}+E_{3}\right)^{2}-\vec{Q}^{2}}, \quad E^{\prime *}=\frac{1}{2} \sqrt{\left(E_{2}^{\prime}+E_{3}^{\prime}\right)^{2}-\vec{Q}^{2}}
$$

Here the energies $E_{2}, E_{3}$ correspond to the nucleons in the initial deuteron. The expressions for them are identical to the ones in the single scattering case and defined by Eq.(29). The primed energies $E_{1}^{\prime}, E_{2}^{\prime}, E_{3}^{\prime}$ are related with the corresponding momenta (Fig.1c) in the standard manner

$E_{1}^{\prime}=\sqrt{m^{2}+\left(\vec{p}+\vec{Q}-\vec{q}^{\prime}-\vec{q}^{\prime \prime}\right)^{2}}, \quad E_{2}^{\prime}=\sqrt{m^{2}+\left(\vec{Q}+\vec{q}^{\prime \prime}\right)^{2}}, \quad E_{3}^{\prime}=\sqrt{m^{2}+\vec{q}^{\prime \prime 2}}$

Applying Eqs.(201)-(21) to describe the transformation of the deuteron wave function due to the Wigner rotation, we can find the directions of the rotation axes for the initial and final deuterons, respectively:

$$
\vec{n}=\frac{\vec{q}^{\prime} \times \vec{Q}}{\left|\vec{q}^{\prime} \times \vec{Q}\right|}, \quad \vec{n}^{\prime}=\frac{\vec{q}^{\prime \prime} \times \vec{Q}}{\left|\vec{q}^{\prime \prime} \times \vec{Q}\right|} .
$$

Note, these axes do not coincide here as it was in the one nucleon exchange and single scattering cases.

The rotation angles are expressed through their tangents, which are defined by Eq.(32) for the initial deuteron, and by the following relations - for the final deuteron:

$$
\begin{aligned}
\operatorname{tg} \frac{\omega_{2}^{\prime}}{2} & =-\frac{\left|\vec{q}^{\prime \prime} \times \vec{Q}\right|}{m\left(E_{2}^{\prime}+E_{3}^{\prime}+2 E^{\prime *}\right)+2 E^{\prime *}\left(E_{2}^{\prime}+E^{\prime *}\right)} \\
\operatorname{tg} \frac{\omega_{3}^{\prime}}{2} & =\frac{\left|\vec{q}^{\prime \prime} \times \vec{Q}\right|}{m\left(E_{2}^{\prime}+E_{3}^{\prime}+2 E^{\prime *}\right)+2 E^{\prime *}\left(E_{3}^{\prime}+E^{\prime *}\right)} .
\end{aligned}
$$

In Eq.(44) we have two $N N$ t-matrices which correspond to two rescattering vertices. There are the off-energy shell matrices defined in the previous subsection. We get $\sqrt{s_{13}}$ 
and $\sqrt{s_{12}^{\prime}}$ as the arguments of the first and second $t$-matrices, correspondingly. The c.m. relative momenta of the colliding and scattering nucleons in the first vertex are related with the nucleon momenta in the deuteron Breit frame by the following expressions:

$$
\begin{aligned}
\vec{k} & =\vec{Q}-\frac{(\vec{p}+\vec{Q})\left(E_{3}+E_{13}^{*}\right)+\vec{q}^{\prime}\left(E_{p}+E_{13}^{*}\right)}{E_{p}+E_{3}+2 E_{13}^{*}} \\
\vec{k}^{\prime} & =\vec{q}^{\prime \prime}-\left(\vec{p}+\vec{Q}-\vec{q}^{\prime}\right) \frac{E_{3}^{\prime}+E_{13}^{\prime *}}{E_{1}^{\prime}+E_{3}^{\prime}+2 E_{13}^{\prime *}} .
\end{aligned}
$$

The arguments of the second NN $t$-matrix are

$$
\begin{aligned}
\vec{\kappa} & =\vec{q}^{\prime \prime}-\left(\vec{p}+\vec{Q}-\vec{q}^{\prime}\right) \frac{E_{2}+E_{1}^{*}}{E_{1}^{\prime}+E_{2}+2 E_{12}^{*}} \\
\vec{\kappa}^{\prime} & =-\vec{Q}-\frac{(\vec{p}+\vec{Q})\left(E_{2}^{\prime}+E_{12}^{\prime *}\right)+\vec{q}^{\prime \prime}\left(E_{p}+E_{12}^{*}\right)}{E_{p}+E_{2}^{\prime}+2 E_{12}^{\prime *}}
\end{aligned}
$$

where the two-nucleons center-of-mass energies can be expressed by means of the corresponding Mandelstam variables $s$ :

$$
\begin{aligned}
& E_{12}^{*}=\sqrt{s_{12}} / 2=\frac{1}{2} \sqrt{\left(E_{1}+E_{2}\right)^{2}-\left(\vec{p}+\vec{Q}-\vec{q}^{\prime \prime}\right)^{2}} \\
& E_{12}^{\prime *}=\sqrt{s_{12}^{\prime}} / 2=\frac{1}{2} \sqrt{\left(E_{p}+E_{2}^{\prime}\right)^{2}-\left(\vec{p}+\vec{Q}-\vec{q}^{\prime \prime}\right)^{2}} \\
& E_{13}^{*}=\sqrt{s_{13}} / 2=\frac{1}{2} \sqrt{\left(E_{p}+E_{3}\right)^{2}-\left(\vec{p}+\vec{Q}-\vec{q}^{\prime}\right)^{2}} \\
& E_{13}^{\prime *}=\sqrt{s_{13}^{\prime}} / 2=\frac{1}{2} \sqrt{\left(E_{1}+E_{3}^{\prime}\right)^{2}-\left(\vec{p}+\vec{Q}-\vec{q}^{\prime}\right)^{2}} .
\end{aligned}
$$

The normalization factors $N$ and $N^{\prime}$ are connected with the transformations of the nucleon-nucleon $t$-matrices as follows:

$$
\begin{gathered}
N=\sqrt{\frac{E_{13}^{*}\left(E_{p}+E_{3}\right)}{2 E_{p} E_{3}}} \sqrt{\frac{E_{13}^{\prime *}\left(E_{1}+E_{3}^{\prime}\right)}{2 E_{1} E_{3}^{\prime}}} \frac{\sqrt{s_{13}}}{E_{p}+E_{3}} \cos \frac{\chi}{2} \cos \frac{\chi_{3}}{2} \cos \frac{\chi_{1}}{2} \cos \frac{\chi_{3}^{\prime}}{2} \\
N^{\prime}=\sqrt{\frac{E_{12}^{*}\left(E_{1}+E_{2}\right)}{2 E_{1} E_{2}} \sqrt{\frac{E_{12}^{\prime *}\left(E_{p}+E_{2}^{\prime}\right)}{2 E_{p} E_{2}^{\prime}}} \frac{\sqrt{s_{12}^{\prime}}}{E_{p}+E_{2}^{\prime}} \cos \frac{\varphi_{1}}{2} \cos \frac{\varphi_{2}}{2} \cos \frac{\varphi}{2} \cos \frac{\varphi_{2}^{\prime}}{2} .}
\end{gathered}
$$

Each of these factors includes the normalization coefficient $\mathcal{N}$ related with the Jacobians of the transformations from the c.m. to the reference frame, Eq.(11), the kinematical factor $F$ defined in Eq.(41), and cosines of the Wigner rotation angles which follow from the Wigner rotation operators, Eq.(12). The expressions for the tangents of the Wigner angles are given in Appendix. 
The three nucleon free propagator in Eq.(44) can be decomposed on the two terms using the well known formula:

$$
\begin{aligned}
\frac{1}{E_{d}+E_{p}-E_{1}^{\prime}-E_{2}^{\prime}-E_{3}^{\prime}+i \varepsilon}= & \mathcal{P} \frac{1}{E_{d}+E_{p}-E_{1}^{\prime}-E_{2}^{\prime}-E_{3}^{\prime}}- \\
& i \pi \delta\left(E_{d}+E_{p}-E_{1}^{\prime}-E_{2}^{\prime}-E_{3}^{\prime}\right) .
\end{aligned}
$$

The principal value part is often neglected to simplify further calculations. However this method is efficient only to evaluate the double scattering contribution. In order to get a more precise result, it is very important to take into account the both terms of the three nucleon propagator. In our calculations we perform all integrations without any simplifications by using standard CERNLIB programs. The difference between the results obtained with and without the principal value part of the free propagator, is discussed below.

\section{Polarization observables}

In the previous section the amplitude of the deuteron- proton elastic scattering has been considered in detail. We have shown how three contributions contained in this amplitude can be expressed in terms of the deuteron wave function and nucleon-nucleon $t$-matrices defined in the deuteron Breit frame.

On the other hand, as in ref. [8], the $d p$ elastic scattering amplitude $\mathcal{J}_{d p \rightarrow d p}$ can be decomposed in spin-1 operators acting in the deuteron spin space and spin- $1 / 2$ operators $\sigma_{i}$ acting in the proton spin space:

$$
\begin{aligned}
\mathcal{J}_{d p \rightarrow d p}=<m^{\prime} \mathcal{M}_{d}^{\prime} \mid & f_{1}+f_{2}(\vec{S} \vec{y})+f_{3} Q_{x x}+f_{4} Q_{y y}+f_{5}(\vec{\sigma} \vec{x})(\vec{S} \vec{x})+ \\
& f_{6}(\vec{\sigma} \vec{x}) Q_{x y}+f_{7}(\vec{\sigma} \vec{y})+f_{8}(\vec{\sigma} \vec{y})(\vec{S} \vec{y})+f_{9}(\vec{\sigma} \vec{y}) Q_{x x}+ \\
& f_{10}(\vec{\sigma} \vec{y}) Q_{y y}+f_{11}(\vec{\sigma} \vec{z})(\vec{S} \vec{z})+f_{12}(\vec{\sigma} \vec{z}) Q_{y z} \mid m \mathcal{M}_{d}>.
\end{aligned}
$$

Due to the time-reversal and parity invariance, we have only 12 linearly independent amplitudes $f_{i}$. These amplitudes can be calculated by using the technique presented 
above. The operators $\sigma_{i}$ are the Pauli matrices, while $S_{i}$ and $Q_{i j}$ are the spin-1 vector and quadrupole operators, respectively:

$$
Q_{i j}=\frac{1}{2}\left(S_{i} S_{j}+S_{j} S_{i}\right)-\frac{2}{3} \delta_{i j} \hat{I}, \quad Q_{x x}+Q_{y y}+Q_{z z}=0
$$

This paper considers only two polarization observables: the vector analyzing power of the deuteron $A_{y}$ and tensor analyzing power $A_{y y}$ which are defined by the standard manner:

$$
A_{y}=\frac{\operatorname{Tr}\left(\mathcal{J} S_{y} \mathcal{J}^{\dagger}\right)}{\operatorname{Tr}\left(\mathcal{J} \mathcal{J}^{\dagger}\right)}, \quad A_{y y}=\frac{\operatorname{Tr}\left(\mathcal{J} Q_{y y} \mathcal{J}^{\dagger}\right)}{\operatorname{Tr}\left(\mathcal{J} \mathcal{J}^{\dagger}\right)}
$$

The squared $d p$-amplitude summarized over all the spin projections can be presented through amplitudes $f_{i}$ as

$$
\begin{aligned}
\operatorname{Tr}\left(\mathcal{J J}^{\dagger}\right)= & 6\left(f_{1}^{2}+f_{7}^{2}\right)+4\left(f_{2}^{2}+f_{5}^{2}+f_{8}^{2}+f_{11}^{2}\right)+\frac{4}{3}\left(f_{3}^{2}+f_{4}^{2}+f_{9}^{2}+f_{10}^{2}\right)+ \\
& \left(f_{6}^{2}+f_{12}^{2}\right)-\frac{4}{3} \operatorname{Re}\left(f_{3} f_{4}^{*}+f_{9} f_{10}^{*}\right) .
\end{aligned}
$$

Using the standard technique one can also obtain traces to define the vector

$$
\begin{array}{r}
\operatorname{Tr}\left(\mathcal{J} S_{y} \mathcal{J}^{\dagger}\right)=\operatorname{Re}\left[8\left(f_{1} f_{2}^{*}+f_{7} f_{8}^{*}\right)-\frac{4}{3}\left(f_{2} f_{3}^{*}+f_{8} f_{9}^{*}\right)+\right. \\
\left.\frac{8}{3}\left(f_{2} f_{4}^{*}+f_{8} f_{10}^{*}\right)+2\left(f_{5} f_{6}^{*}+f_{11} f_{12}^{*}\right)\right]
\end{array}
$$

and tensor analyzing powers

$$
\begin{aligned}
\operatorname{Tr}\left(\mathcal{J} Q_{y y} \mathcal{J}^{\dagger}\right)= & \frac{4}{3}\left(f_{2}^{2}+f_{8}^{2}\right)+\frac{2}{9}\left(f_{3}^{2}+f_{9}^{2}\right)-\frac{4}{9}\left(f_{4}^{2}+f_{10}^{2}\right)-\frac{2}{3}\left(f_{5}^{2}+f_{11}^{2}\right)- \\
& \frac{1}{6}\left(f_{6}^{2}+f_{12}^{2}\right)+\frac{8}{3} \operatorname{Re}\left(f_{1} f_{4}^{*}+f_{7} f_{10}^{*}\right)-\frac{4}{3} \operatorname{Re}\left(f_{1} f_{3}^{*}+f_{7} f_{9}^{*}\right)+ \\
& \frac{4}{9} \operatorname{Re}\left(f_{3} f_{4}^{*}+f_{9} f_{10}^{*}\right) .
\end{aligned}
$$

In general, we have to apply the spin transformation technique to relate the laboratory observables to the Breit frame ones. However, we have considered only those observables which are defined by the polarization along the normal to the scattering plane. In this case the definitions of the polarization observables are identical both - for the laboratory and Breit frames. It is not the case for other observables, for example, $A_{x x}, A_{x z}$, etc. 


\section{Results and discussions}

The results of our calculations are presented in Figs.(2-5). We have performed our investigations at two deuteron kinetic energies: $395 \mathrm{MeV}$ and $1200 \mathrm{MeV}$. This choice was motivated by two reasons. First, there are sets of experimental data at these energies [1]-[3]. Second, these energies have demonstrated the range, where the method presented here can be applied.

The dashed curves in Figs. (2-5) correspond to the calculations which include only the one nucleon exchange and single scattering (ONE+SS) contributions. The solid line is the result which also takes the double scattering (DS) into account. All the calculations have been carried out with the CD Bonn deuteron wave function [11]. We have obtained a good agreement of the theoretical curves with the experimental data at the deuteron kinetic energy of $395 \mathrm{MeV}$. Note, the contribution of the double scattering is not very big at this energy, especially for $A_{y y}$.

The other situation has been revealed for the higher energy. An agreement between the obtained curves and experimental data at the deuteron energy of $1200 \mathrm{MeV}$ is not so obvious. The vector analyzing power is very well described up to the angle of $90^{0}$ c.m., while the behaviour of the tensor analyzing power is reproduced only qualitatively in a wide angular range. Here the single scattering contribution dominates only up to $\theta^{*}=30^{\circ}$, whereas the both polarization observables are very sensitive at this energy to the double scattering at larger angles. Especially the behaviour of the $A_{y y}$ changes very strongly due to the DS term having been taken into account. On the contrary, at the deuteron energy of $395 \mathrm{MeV}$ the double scattering contribution is practically insignificant for the tensor analyzing power but for the vector analyzing power it is up to $50 \%$.

The results of the calculations with and without the principal value part of the threenucleon free propagator are given in Figs.(6-9). The curves corresponding to the predictions for the deuteron energy of $395 \mathrm{MeV}$, are practically undistinguished. It is a rather clear result, since the double scattering contribution is not so large at this energy.

At $1200 \mathrm{MeV}$ the contribution of the principal value part of the free propagator is more 
significant. Note, that the full calculation describes the vector analyzing power better than the simplified method, especially at the $40^{0}-80^{0}$ c.m. angles, where the double scattering is very important. But it is not the case for the tensor analyzing power. Here the full calculation gives the smaller absolute value as compared with the experimental data in the range, where $A_{y y}$ has a maximum or minimum, while the result obtained by the reduced method is in a good agreement with the data. However, it is necessary to keep in mind, that this shortage is observed even in the range, where the single scattering dominates $\left(\theta^{*} \leq 40^{0}\right)$. From the above it is possible to conclude, that the reason of this deficiency is not related with the three nucleon free propagator in the double scattering term.

It should be noted that these observables are also sensitive to the nucleon-nucleon amplitudes. Unfortunately, to describe $N N$ interactions we have used the model offered twenty years ago, when the nucleon-nucleon phase shift analysis was incomplete. Besides, up to now we have no reliable data of the $n p$ amplitudes at the nucleon kinetic energy higher than $1.3 \mathrm{GeV}$ [41] and that is very important to study reactions with light nuclei at intermediate energies. Maybe, that the qualitative disagreement between the theoretical predictions and experimental data at the deuteron energy of $1200 \mathrm{MeV}$ is caused by incomplete $N N$ interaction description. In this connection the modern parameterization of the nucleon-nucleon $t$-matrix in a wide energy range is needed.

\section{Conclusion}

In this paper we have presented a method to calculate the amplitude of the deuteronproton elastic scattering at intermediate energies. Special attention was given to the questions connected with the relativistic effects. The transformation of the deuteron

wave function in the rest frame to a moving system was performed, that allowed us to use the nonrelativistic DWF at rather high energies. In order to describe nucleon-nucleon interactions in a wide energy range, we have used parameterization of the $N N t$-matrix. The spin transformation technique has been also applied to relate this $t$-matrix given in the c.m. to that in the reference frame. 
Using the method presented here we have managed to describe the experimental data on the vector, $A_{y}$, and tensor, $A_{y y}$, analyzing powers at two energies. We got a good agreement between our predictions and experimental data at the deuteron kinetic energy of $395 \mathrm{MeV}$ and acceptable agreement - at $1200 \mathrm{MeV}$. We believe that the difference between the obtained predictions and data is related with the shortcomings of the nucleonnucleon parameterization. It is possible that the deuteron-proton scattering description can be improved, if the modern $N N$ model is used.

In the future it is planned to apply the approach developed in this paper to describe experimental data at other energies. In particular, the data obtained at the Nuclotron (Dubna) at $880 \mathrm{MeV}$ of the deuteron energy [42] can be described in this formalism.

Acknowledgements: The author is grateful to Dr. V.P. Ladygin for fruitful discussions. This work has been supported by the Russian Foundation for Basic Research under grant $N^{0}$ 07-02-00102a.

\section{Appendix}

Applying Eq.(12) we can obtain the expressions for the Wigner angles. For the first nucleon-nucleon scattering we have

$$
\begin{aligned}
\operatorname{tg} \frac{\chi}{2} & =\frac{\left|\vec{p} \times \vec{p}_{3}\right|}{m\left(E_{p}+E_{3}+2 E_{13}^{*}\right)+2 E_{13}^{*}\left(E_{p}+E_{13}^{*}\right)} \\
\operatorname{tg} \frac{\chi_{3}}{2} & =\frac{\left|\vec{p} \times \vec{p}_{3}\right|}{m\left(E_{p}+E_{3}+2 E_{13}^{*}\right)+2 E_{13}^{*}\left(E_{3}+E_{13}^{*}\right)} \\
\operatorname{tg} \frac{\chi_{1}}{2} & =\frac{\left|\vec{p}_{1} \times \vec{q}^{\prime \prime}\right|}{m\left(E_{1}+E_{3}^{\prime}+2 E_{13}^{\prime *}\right)+2 E_{13}^{\prime *}\left(E_{1}+E_{13}^{\prime *}\right)} \\
\operatorname{tg} \frac{\chi_{3}^{\prime}}{2} & =\frac{\left|\vec{p}_{1} \times \vec{q}^{\prime \prime}\right|}{m\left(E_{1}+E_{3}^{\prime}+2 E_{13}^{\prime *}\right)+2 E_{13}^{\prime *}\left(E_{3}^{\prime}+E_{13}^{\prime *}\right)},
\end{aligned}
$$

where $\chi, \chi_{3}$ are the angles corresponding to the beam proton and struck nucleon from the deuteron, respectively, and $\chi_{1}, \chi_{3}^{\prime}$ are the angles corresponding to the scattered nucleons (Fig. 1c). 
The analogous expressions are obtained for the second scattering vertex:

$$
\begin{aligned}
\operatorname{tg} \frac{\varphi_{1}}{2} & =\frac{\left|\vec{p}_{1} \times \vec{q}^{\prime}\right|}{m\left(E_{1}+E_{2}+2 E_{12}^{*}\right)+2 E_{12}^{*}\left(E_{1}+E_{12}^{*}\right)} \\
\operatorname{tg} \frac{\varphi_{2}}{2} & =\frac{\left|\vec{p}_{1} \times \vec{q}^{\prime}\right|}{m\left(E_{1}+E_{2}+2 E_{12}^{*}\right)+2 E_{12}^{*}\left(E_{2}+E_{12}^{*}\right)} \\
\operatorname{tg} \frac{\varphi}{2} & =\frac{\left|\vec{p}^{\prime} \times \vec{p}_{2}^{\prime}\right|}{m\left(E_{p}+E_{2}^{\prime}+2 E_{12}^{\prime *}\right)+2 E_{12}^{\prime *}\left(E_{p}+E_{12}^{\prime *}\right)} \\
\operatorname{tg} \frac{\varphi_{2}^{\prime}}{2} & =\frac{\left|\vec{p}^{\prime} \times \vec{p}_{2}^{\prime}\right|}{m\left(E_{p}+E_{2}^{\prime}+2 E_{12}^{\prime *}\right)+2 E_{12}^{\prime *}\left(E_{2}^{\prime}+E_{12}^{\prime *}\right)} .
\end{aligned}
$$

Here $\varphi_{1}, \varphi_{2}$ correspond to the colliding nucleons, while $\varphi, \varphi_{2}^{\prime}$ correspond to the scattered proton and nucleon from the deuteron, respectively.

\section{References}

[1] M.Garcon et al., Nucl.Phys. A458, 287 (1986)

[2] J.Arvieux et al., Nucl.Phys. A431, 613 (1984)

[3] M.Haji-Saied et al., Phys. Rev. C36, 2010 (1987)

[4] Sun Tsu-hsun et al. Phys.Rev. C31 515 (1985)

[5] A.Rahbar et al. Rhys. Lett B194 338 (1987)

[6] V.Ghazikhanian et al. Phys.Rev. C43 1532 (1991)

[7] W.Glöckle, et al., Phys.Rep. 274, 110 (1996)

[8] G.Alberi, M.Bleszynski, T.Jaroszewicz: Ann. Phys. 142, 299 (1982)

[9] M.Bleszynski et.al., Phys.Lett. B106, 42 (1981)

[10] R.Machleidt, K.Holinde, Ch.Elster, Phys.Rep. 149, 1 (1987).

[11] Machleidt, R.: Phys. Rev. C63, 024001 (2001)

[12] Lacombe, M., et al.: Phys. Rev. C21, 861 (1980) 
[13] V.G.J.Stoks, et al., Phys.Rev. C49, 2950 (1994)

[14] R.B.Wiringa, V.G.J.Stoks, and R.Schiavilla, Phys.Rev. C51, 38 (1995)

[15] T.S.H.Lee, Phys.Rev. C29, 195 (1984)

[16] E.E. van Faassen and J.A.Tjon, Phys.Rev. C30, 285 (1984)

[17] Ch.Elster and P.C.Tandy, Phys.Rev. C40, 881 (1989)

[18] V.I.Kukulin, et al., J.Phys.G: Nucl.Part.Phys. 27, 1852 (2001)

[19] V.A.Knyr, V.G.Neudatchin, N.A.Khokhlov, Phys.Atom.Nucl. 69, 2034 (2006)

[20] R.Fong, J.Sucher, Jour.Math.Phys. 5, 456 (1964)

[21] P.A.M.Dirac, Rev.Mod.Phys. 21, 392 (1949)

[22] E.P.Wigner, Nuovo Cim. 3, 517 (1956)

[23] L.L.Foldy, Phys.Rev. 122, 275 (1961)

[24] B.D.Keister and W.N.Polyzou, Adv.Nucl.Phys., 20, 225 (1991)

[25] H.Witala, et al., Phys.Rev.C71, 054001 (2005)

[26] T.Lin, et al., nucl-th/0702005 (2007)

[27] W.W.Buck and F.Gross, Phys.Rev. D20, 2361 (1979)

[28] B.D.Keister and J.A.Tjon, Phys.Rev. C26, 578 (1982)

[29] L.P.Kaptari, et al., Phys.Rev. C57, 1097 (1998)

[30] M. Lacombe et al., Phys.Lett. B101, 139 (1981)

[31] V.A.Karmanov, Nucl.Phys.A362, 331 (1981)

[32] V.A.Karmanov, Phys.Elem. Chast.Atom.Yadra 19, 525 (1988) 
[33] J.Carbonell and V.A.Karmanov, Nucl.Phys. A581, 625 (1994)

[34] Love, W. G., Franey. M. A.: Phys. Rev. C24, 1073 (1981); Love, W.G., Franey, M.A.: ibid. C31, 488 (1985)

[35] E.O.Alt, P.Grassberger, W.Sandhas, Nucl.Phys. B2, 167 (1967)

[36] E.Schmid, H.Ziegelmann, The Quantum Mechanical Three-Body Problem (Oxford, Pergamon Press, 1974)

[37] V.I. Ritus, JETP 40, 352 (1961)

[38] N.B.Ladygina, A.V.Shebeko, Few Body Syst.33, 49 (2003)

[39] N.B.Ladygina, A.V.Shebeko, Eur.Phys.J. A22, 29 (2004)

[40] H. Garcilazo, Phys. Rev. C16, 1996 (1976)

[41] http://gwdac.phys.gwu.edu

[42] P.K. Kurilkin et al., Proc. of the 18-th Int. Baldin seminar on high energy physics problems "Relativistic nuclear Physics \& quantum chromodynamics" 25-30 Sept., 2006, Dubna, Russia, to be published; K.Suda et al., Proc. of the 17-th International Spin Symposium SPIN2006, 2-7 Oct., 2006, Kyoto, Japan, to be published. 


\section{Figure captions}

Fig.1 The diagrams included into consideration. (a) The one nucleon exchange diagram. (b) The single scattering diagram. (c) The double scattering diagram.

Fig.2 The deuteron vector analyzing power at $395 \mathrm{MeV}$ as a function of the c.m. angle, the data are taken from ref. [1]. The dashed line corresponds to the ONE+SS, the solid line shows the ONE+SS+DS calculation.

Fig.3 The deuteron tensor analyzing power at $395 \mathrm{MeV}$ as a function of the c.m. angle, the data are taken from ref. [1]. The dashed line corresponds to the ONE+SS, the solid line shows the ONE+SS+DS calculation.

Fig.4 The deuteron vector analyzing power at $1200 \mathrm{MeV}$ as a function of the c.m. angle, the data are taken: $(\bullet)$ from ref. [2], $(\triangle)$ from ref. [3]. The dashed line corresponds to the ONE+SS, the solid line shows the ONE+SS+DS calculation.

Fig.5 The deuteron tensor analyzing power at $1200 \mathrm{MeV}$ as a function of the c.m. angle, the data are taken: $(\bullet)$ from ref. [2], $(\triangle)$ from ref. [3]. The dashed line corresponds to the $\mathrm{ONE}+\mathrm{SS}$, the solid line shows the $\mathrm{ONE}+\mathrm{SS}+\mathrm{DS}$ calculation.

Fig.6 The deuteron vector analyzing power at $395 \mathrm{MeV}$ as a function of the c.m. angle, the data are taken from ref. [1]. The solid and dashed lines correspond to the calculations with and without the principal value part of the propagator.

Fig.7 The deuteron tensor analyzing power at $395 \mathrm{MeV}$ as a function of the c.m. 
angle, the data are taken from ref. [1]. The solid and dashed lines correspond to the calculations with and without the principal value part of the propagator.

Fig.8 The deuteron vector analyzing power at $1200 \mathrm{MeV}$ as a function of the c.m. angle, the data are taken: $(\bullet)$ from ref. [2], $(\triangle)$ from ref. [3]. The solid and dashed lines correspond to the calculations with and without the principal value part of the propagator.

Fig.9 The deuteron tensor analyzing power at $1200 \mathrm{MeV}$ as a function of the c.m. angle, the data are taken: $(\bullet)$ from ref. [2], $\triangle$ ) from ref. [3]. The solid and dashed lines correspond to the calculations with and without the principal value part of the propagator. 


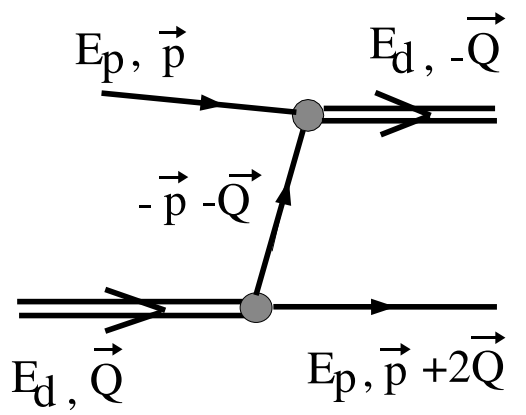

(a)

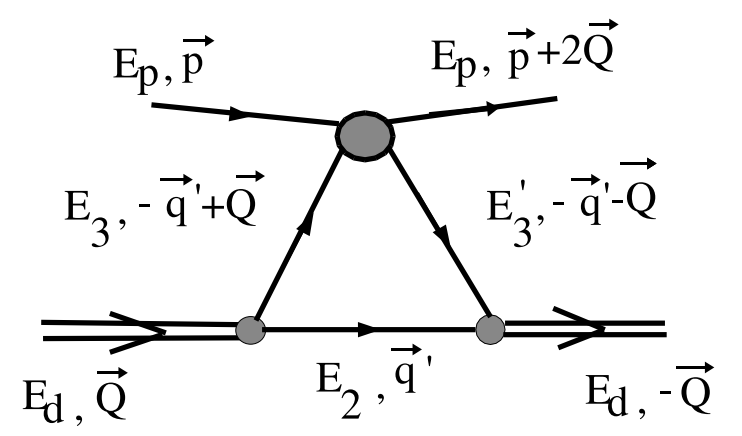

(b)

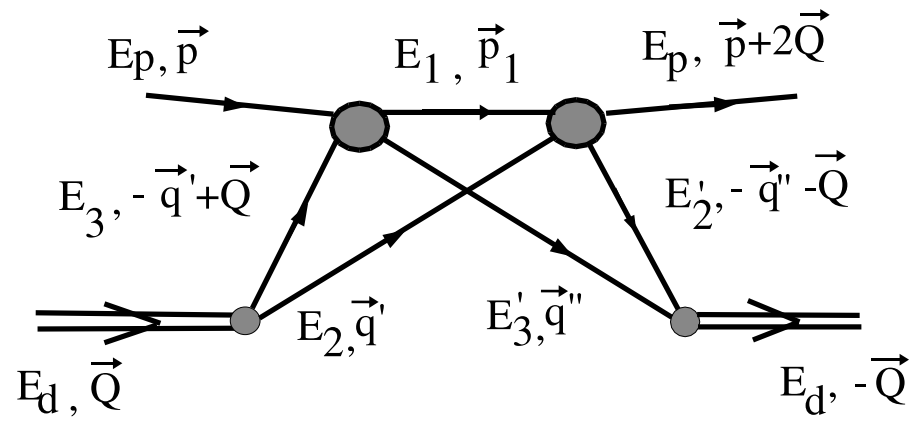

(c)

Figure 1: Fig.1 


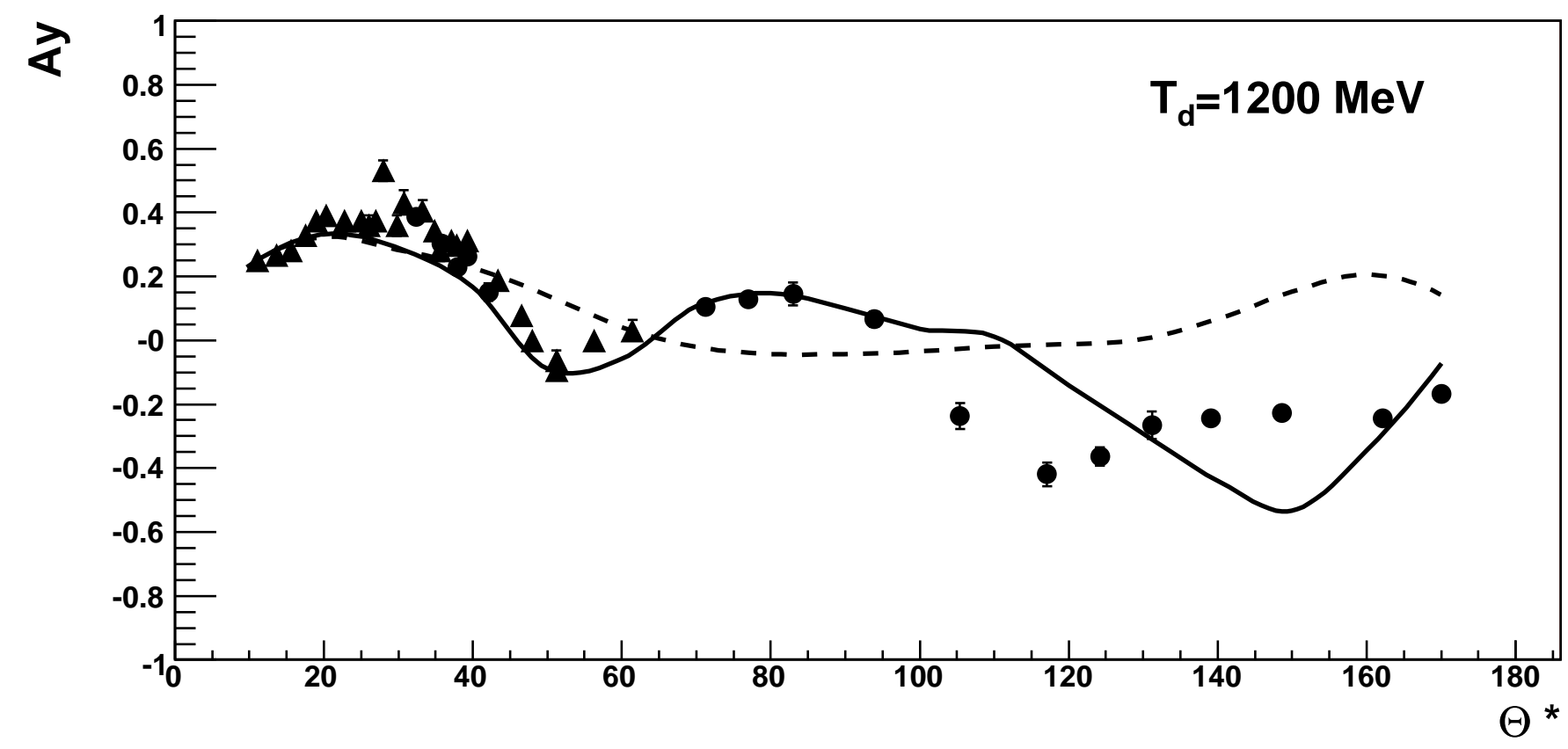

Figure 4: Fig.4

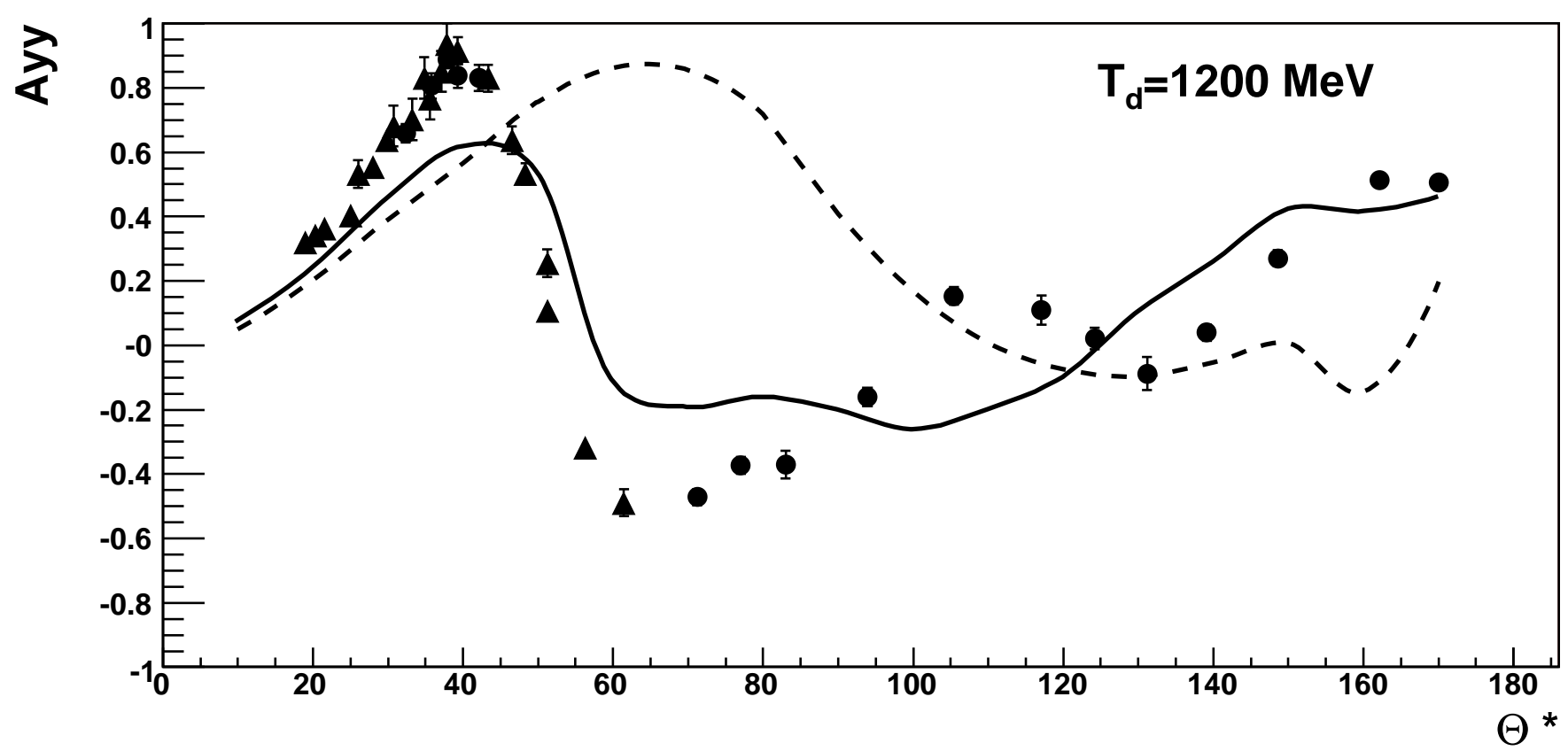

Figure 5: Fig.5 


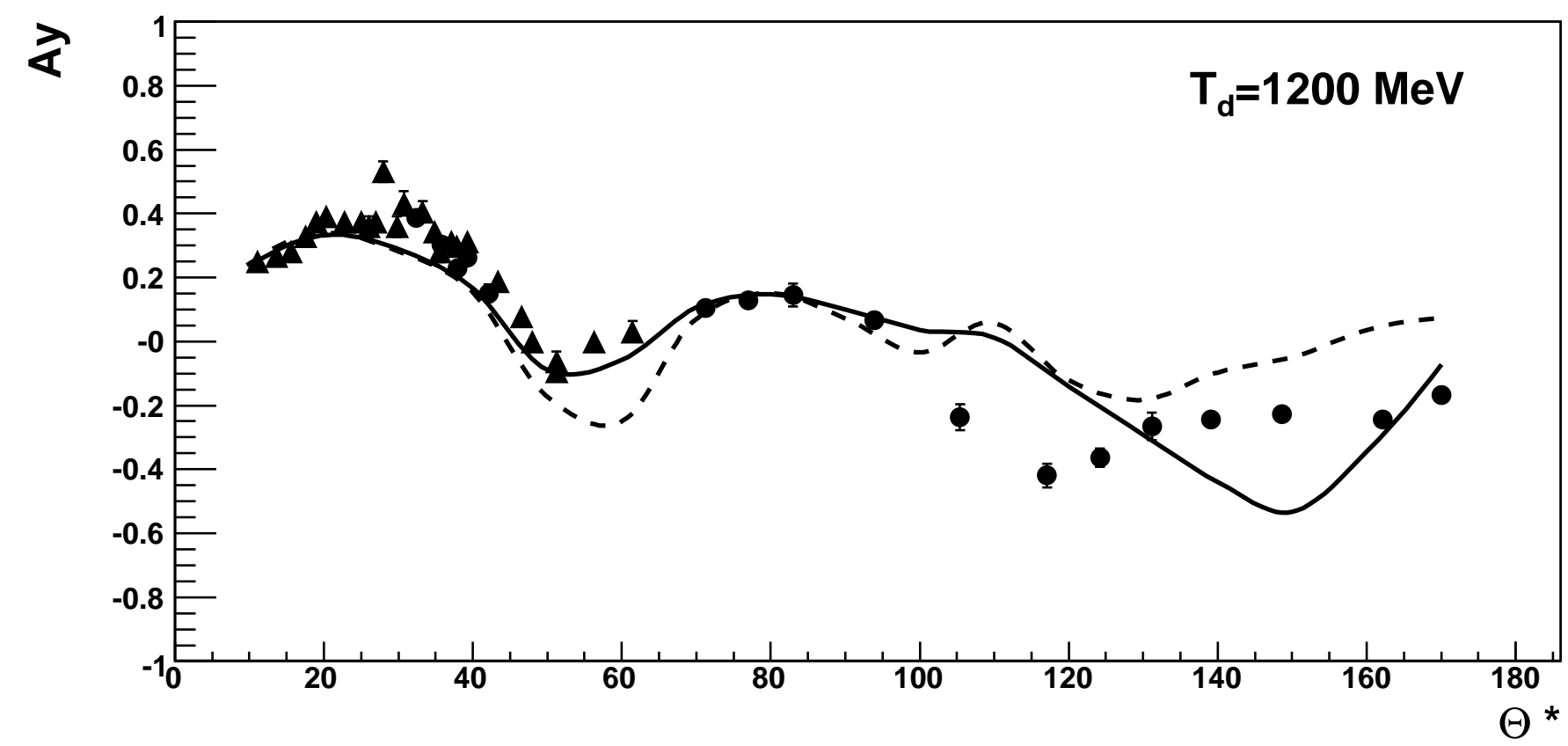

Figure 8: Fig.8

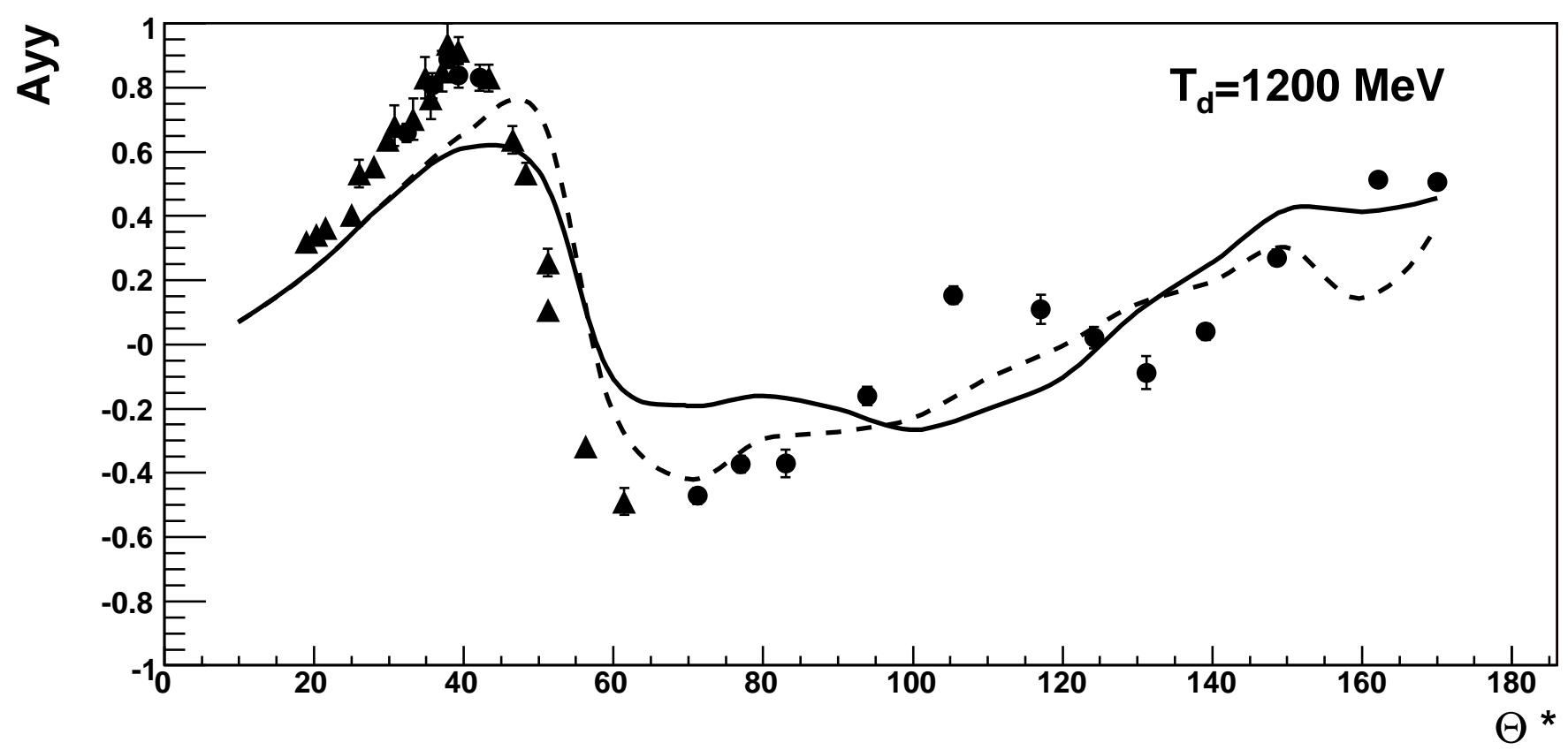

Figure 9: Fig.9 